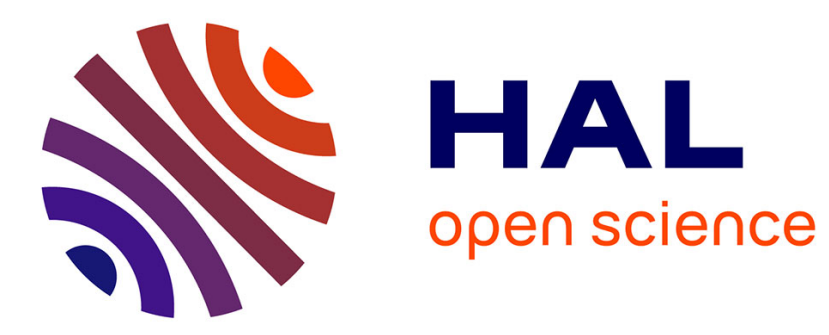

\title{
Foam on troubled water: Capillary induced finite-time arrest of sloshing waves
}

\author{
Francesco Viola, P.-T Brun, Benjamin Dollet, François Gallaire
}

\section{To cite this version:}

Francesco Viola, P.-T Brun, Benjamin Dollet, François Gallaire. Foam on troubled water: Capillary induced finite-time arrest of sloshing waves. Physics of Fluids, 2016, 28 (9), pp.091701. 10.1063/1.4961260 . hal-01367501

\section{HAL Id: hal-01367501 \\ https://hal.science/hal-01367501}

Submitted on 16 Sep 2016

HAL is a multi-disciplinary open access archive for the deposit and dissemination of scientific research documents, whether they are published or not. The documents may come from teaching and research institutions in France or abroad, or from public or private research centers.
L'archive ouverte pluridisciplinaire HAL, est destinée au dépôt et à la diffusion de documents scientifiques de niveau recherche, publiés ou non, émanant des établissements d'enseignement et de recherche français ou étrangers, des laboratoires publics ou privés. 


\title{
Foam on troubled water: capillary induced finite-time arrest of sloshing waves
}

\author{
Francesco Viola, ${ }^{1}$ P.-T. Brun, ${ }^{2}$ Benjamin Dollet, ${ }^{3}$ and François Gallaire ${ }^{1, a)}$ \\ 1) Laboratory of Fluid Mechanics and Instabilities, EPFL, 1015 Lausanne, \\ Switzerland \\ ${ }^{2)}$ Department of Mathematics, Massachusetts Institute of Technology, Cambridge, \\ Massachusetts 02139, USA \\ 3) Institut de Physique de Rennes, UMR CNRS 6251, Université Rennes 1 Bâtiment 11A, \\ Campus de Beaulieu, 35042 Rennes Cedex, France
}

(Dated: 6 August 2016)

Interfacial forces exceed gravitational forces on a scale small relative to the capillary length - two millimeters in the case of an air-water interface - and therefore dominate the physics of sub-millimetric systems. They are of paramount importance for various biological taxa and engineering processes where the motion of a liquid meniscus induces a viscous frictional force that exhibits a sublinear dependence in the meniscus velocity, i.e. a power law with an exponent smaller than one. Interested in the fundamental implications of this dependence, we use a liquid-foam sloshing system as a prototype to exacerbate the effect of sublinear friction on the macroscopic mechanics of multi-phase flows. In contrast to classical theory, we uncover the existence of a finite-time singularity in our system yielding the arrest of the fluid's oscillations. We propose a minimal theoretical framework to capture this effect, thereby amending the paradigmatic damped harmonic oscillator model. Our results suggest that, although often not considered at the macroscale, sublinear capillary forces govern the friction at liquid-solid and liquid-liquid interfaces.

\section{INTRODUCTION}

No one who has glimpsed at Hokusai's iconic wave paintings can fail to notice the truthful yet stylized rendering of the singular nature of such multi-scale flows. On a theoretical point of view, overturning, breaking and atomization are supported by nonlinear terms in the equations prescribing the waves' dynamics at large amplitude, so that finding their exact solution is often arduous. Nonlinearities, however, usually become of negligible relative importance for sufficiently low amplitudes of motion, thereby enabling perturbative linearized approaches ${ }^{1}$. These theoretical linear problems are easier to solve than their nonlinear counterparts and have been extremely successful at predicting the stability, characteristic frequencies and growth rates of a wide range of physical systems arising in natural and industrial settings, a classic example of which is the sloshing of fluid in a container ${ }^{2}$. In such a case, the excess of gravitational potential energy associated to the deformation of the free surface is progressively relaxed when periodically turned into kinetic energy and damped by viscous forces ${ }^{2,3}$. In the small amplitude regime, the fundamental sloshing frequency for a cylindrical container is known to be $\omega_{0}=\sqrt{1.84 \mathrm{~g} / R}$ where $g$ is the acceleration of gravity and $R$ the container radius ${ }^{2}$. The classical contributions to the viscous damping rate, $\sigma$, have been identified as the dissipation in the oscillating boundary layers near the container walls and the free surface, and the bulk dissipation ${ }^{3}$. Those dissipative forces have different scalings with respect to the governing parameters (radius $R$, viscosity $\mu$, density $\rho$, gravity $g$ ), but all depend linearly on the free surface velocity ${ }^{2,3}$.

Here, we study the effect of a small modification to this classical problem, as we place a thin layer of foam on a volume of water (see Fig. 1), and explore the dramatic change

\footnotetext{
${ }^{a)}$ Electronic mail: francois.gallaire@epfl.ch
} 
A.

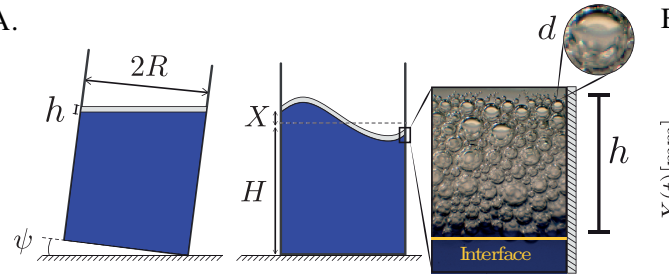

B.

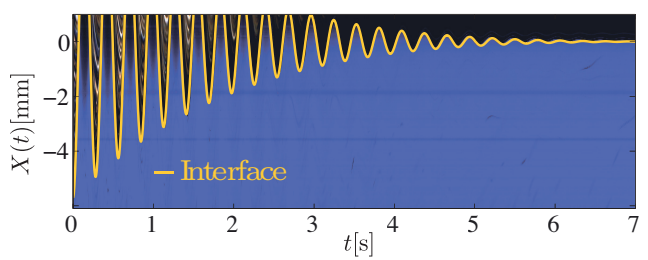

FIG. 1. (A) Experimental setup: a cylindrical container is tilted by an angle $\psi$ to initiate the sloshing of a volume of water surmounted by foam. The foam layer of thickness $h \ll H$ and $h \ll R$ comprises bubbles of typical radius $d$. (B) Shown is the spatio-temporal view of the water-foam contact line $X(t)$ captured in a typical experiment.

in its mechanistic properties. It has been recently shown ${ }^{4}$ that sloshing in a rectangular basin could be strongly damped by the addition of a shallow foam layer at the top. While foam is a complex media with visco-elasto-plastic rheological properties ${ }^{5}$, this significant attenuation was attributed to friction between the foam and the wall, which dominates the dissipation in the foam bulk ${ }^{4}$. In this work, we focus our attention on the nonlinear nature of the dissipation occurring in the foam layer. This source of dissipation emanates from the relative displacement of the liquid channels separating the foam bubbles, known as Plateau borders ${ }^{5}$. The motion of these multiple air-liquid interfaces along the container results in a frictional force that scales nonlinearly with their sliding velocity. Indeed, this force scales like $C a^{2 / 3}$, where $C a$ is the capillary number based on the sliding velocity ${ }^{5-7}$. Owing to their sublinearity (the exponent on the velocity is smaller than one), these frictional terms are found to dominate all linear contributions for small enough values of $\mathrm{Ca}$, which naturally arise for small amplitudes of motion. Therefore, unlike nonlinearities with exponents greater than one, they cannot be neglected, thereby ruining all linear theoretical approaches. Unlike the picture of a linearly damped oscillator classically used in textbooks to model sloshing, we show experimentally and theoretically that the sloshing of the liquid phase does not relax exponentially. The damping rate in fact increases catastrophically at small amplitude and yields a finite-time arrest of the fluid's motion.

\section{EXPERIMENTS}

The setup consists of a cylindrical Plexiglas container of internal radius $R=37.05 \mathrm{~mm}$ that is sealed to an aluminum plate connected itself to a hinge. The container is filled with a column of water of height $H$ which is surmounted by a foam layer of thickness $h$, see Figure 1A. The foam is produced when injecting a commercial hand soap (Hypron $\mathrm{SA}$ ) through a nozzle with a grid with pore size $0.2 \mathrm{~mm}$ and has typical bubbles diameter $d=0.25 \mathrm{~mm}$. The foam's liquid fraction is measured to be $25 \%$ once poured on top of the water column (evaluated using the weight of a volume of foam). This large value relative to dry foams, is due to capillary forces yielding an intake of water effectively enlarging the foam Plateau borders ${ }^{5}$. In the following, the liquid fraction across the foam layer, whose thickness ranges from $h=2.5 \mathrm{~mm}$ to $6.5 \mathrm{~mm}$, is assumed uniform. Using an oscillating drop tensiometer (Tracker, Teclis), we measured the surface tension, $\gamma=31.5 \mathrm{mN} / \mathrm{m}$, of the soap solution. Its viscoelastic surface dilatational modulus, quantifying the relative variation of surface tension upon dilatation and compression, is found below resolution $(0.1 \mathrm{mN} / \mathrm{m})$, showing that the interface can be considered as mobile ${ }^{7}$ and that free shear boundary conditions apply.

Experiments are initiated by tilting the container of an angle $\psi$ and then releasing it, see Fig. 1A. The dynamics following the impact is recorded with a high-speed camera (Phantom Micro M310) operating at $300 \mathrm{~Hz}$. In order to investigate the waves dynamics at small scales, we coupled a Nikon $50 \mathrm{~mm}$ lens with an optical bellows obtaining a resolution of $2 \cdot 10^{-2} \mathrm{~mm}$ 

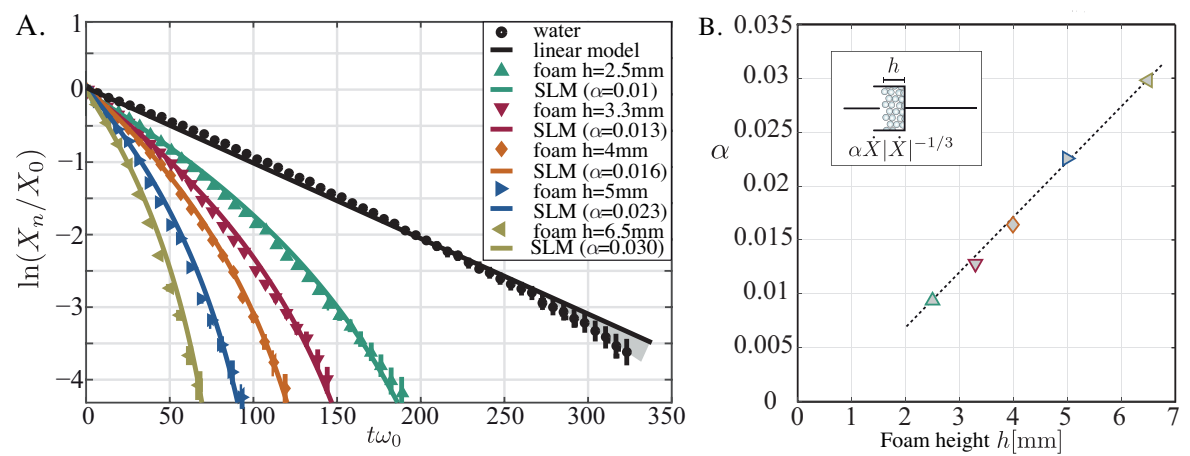

FIG. 2. (A) Logarithmic decrement, $\mathcal{D}=\ln \left(X_{n} / X_{0}\right)$, of the sloshing amplitude for foam thicknesses ranging across values $h=0,2.5,3.3,4,5,6.5 \mathrm{~mm}$. Symbols indicate measurements, while continuous lines represent the envelope solutions of the sublinear model (SLM), with a single fitting parameter $\alpha$ (see $a(t)$ in eq. 5) . (B) Shown is the dependance of the friction force exerted by the foam layer, $F_{f}$, through the friction coefficient $\alpha$ as found in experiments. $F_{f}$ increases linearly with the foam thickness $h$, i.e. with the number of layers of oscillating bubbles.

per pixel on the water-foam interface displacement. After a short transient regime, the flow response was found to exhibit a single mode of oscillation (see Fig. 1B). The frequency of oscillations, $3.50 \mathrm{~Hz}$, matches the theoretical inviscid prediction $\omega_{0}=\sqrt{1.84 g / R}$ within $1 \%$. This result was expected for $h=0$ as the deep water condition $H>2 R$ is satisfied and the container size is well above the capillary length ${ }^{1}$, i.e. $\gamma / \rho g R^{2} \ll 1$. This frequency is found unchanged when adding foam $(0<h<6.5 \mathrm{~mm})$, as already observed in previous experiments ${ }^{4}$ and discussed in section III B.

The attenuation of the gravity waves is investigated by recording the motion $X(t)$ of the water-foam interface at the azimuth corresponding to the largest wave oscillation. Using the function 'orthogonal view' in ImageJ, we follow the evolution of a pixel line over time, thereby reconstituting the oscillatory motion $X(t)$ of the contact line at a given location, see Fig. $1 B$. The natural $\operatorname{logarithm} \mathcal{D}=\ln \left(X_{n} / X_{0}\right)$ of the ratio between the amplitude of the $n$th peak, $X_{n}$, and the amplitude of the first peak, systematically chosen to $X_{0}=5 \mathrm{~mm}$, is used as an instantaneous measure of the growth rate. For the case $h=0$ (no foam), we recover the classical result of a constant damping rate. The logarithmic decrement is linear in time for most of the dynamics, $\mathcal{D} \propto-\sigma \omega_{0} t$, as indicated in Fig. $2 A$ (see section IV for a rationalization of the shaded area). We found $\sigma=0.0098$, which exceeds the theoretical expression $^{3}$ of the viscous damping rate by a factor 2 . This result is in line with other experimental measurements in the literature ${ }^{8}$ and is commonly attributed to the advancing and receding of the dynamic contact line $e^{2}$. As soon as a thin foam layer is added in the system, $\mathcal{D}$ is no longer linear, as shown in Fig. $2 A$ for values of $h$ ranging from $2.5 \mathrm{~mm}$ to $6.5 \mathrm{~mm}$. We find $\mathcal{D}$ to be concave down and negatively correlated to $h$. In other words, the damping rate is greater with foam as evidenced by previous experiments ${ }^{4}$ but also evidently increases as the amplitude of oscillations decreases. In particular this result translates into the fact that the interface does not relax exponentially at a given rate, but instead its decay is progressively hastened. This novel observation suggests the existence of a mechanism submitting smaller oscillations to a stronger damping, that we aim to rationalize.

\section{A NONLINEAR FLUID OSCILLATOR}

\section{A. Equivalent mechanical system}

From this point onward, we restrict ourselves to thin layers of foam and we assume the entire layer of height $h$ is oscillating along with the liquid phase. We model the total 


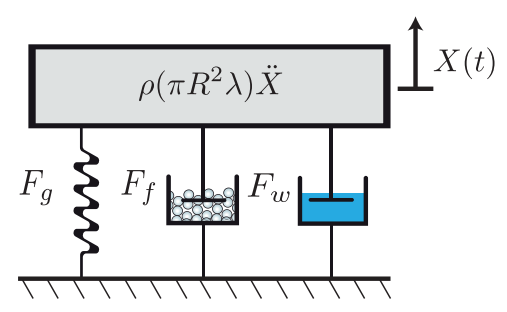

FIG. 3. A sketch of our reduced model for sloshing. The effects of foam are accounted by the sublinear term $F_{f} \propto \dot{X}|\dot{X}|^{-1 / 3}$. This term amends the classical damped oscillator model where the inertial term is balanced by the restoring force, $F_{g} \propto X$, and the viscous damping, $F_{w} \propto \dot{X}$.

oscillating mass as $\pi R^{2}\left(\rho \lambda+\rho_{f} h\right)$, where $\lambda \sim R$ is the penetration length of the wave ${ }^{1}$ and $\rho$ and $\rho_{f}$ denote the density of water and foam, respectively. Given the physical parameters of the problem we place ourselves in the limit $\rho_{f} h \ll \rho \lambda$, i.e. we neglect foam's inertia relative to the one of water. Similarly, the restoring gravitational force acting on this volume of fluid may be estimated considering solely the liquid phase: $F_{g} \sim \rho g \pi R^{2} X$, where $X$ is the amplitude of the water-foam interface and $g$ the acceleration of gravity. In absence of foam, according to the seminal work of Case \& Parkinson $^{3}$, three main dissipation sources exist, they acting: (i) at the free surface, (ii) in the fluid bulk and (iii) in the Stokes boundary layer close to the wall. The last contribution has been shown to dominate the other ones ${ }^{3}$. Denoting by $\mu$ and $\nu$ the water dynamic and kinematic viscosities, and by $\dot{X}$ the oscillation speed, we model the viscous dissipation as a single force $F_{w} \sim 2 \pi R \lambda \mu \dot{X} / \delta$, where $\delta \sim \sqrt{\nu / \omega_{0}}$ is the Stokes layer thickness ${ }^{3}$.

We now turn to model the force $F_{f}$ associated with capillary effects in the foam layer. Let us consider a single wall Plateau border, i.e. the edge separating neighboring bubbles at the wall, sliding along the container vertical wall with velocity $\dot{X}$. Damping of its motion originates from the transition region between the wetting film at the wall and the moving wall Plateau border ${ }^{5}$. The matching between the outer meniscus and the transition zone, involving a balance between viscous and capillary forces, defines the friction force per unit length of a wall Plateau border scaling as $\gamma \mathrm{Ca}^{2 / 3}$, where $\mathrm{Ca}=\mu \dot{X} / \gamma$ is the capillary number and $\gamma$ denotes the surface tension ${ }^{5}$. The total length of surface Plateau borders is given by their typical length, $d$, times the number of Plateau borders in contact with the container that scales as $2 \pi R h / d^{2}$. Thus, the friction force acting at the wall in the foam layer reads

$$
F_{f} \sim \frac{h}{d} R \gamma^{1 / 3} \mu^{2 / 3} \dot{X}|\dot{X}|^{-1 / 3}
$$

where the absolute value is introduced to conveniently treat negative speeds. This result is reminiscent to the force acting on a plate pulled out of a bath ${ }^{6,9}$ and is valid for a mobile interface, i.e. an interface where a free shear boundary condition applies. This point has been verified for the foam used in our experiments (see section II).

Using the estimation for the volume of fluid effectively oscillating and the forces acting on it, we write Newton's $2^{\text {nd }}$ law:

$$
\pi R^{2}(\rho \lambda) \ddot{X}=F_{g}+F_{w}+F_{f} .
$$

Equation (2) constitutes a nonlinear oscillator where inertia is resisted by the restoring force of gravity and the two dissipative terms, $F_{w}$ and $F_{f}$ (see the equivalent mechanical system depicted in Figure 3). We note that $F_{w}$ varies linearly with $\dot{X}$, while $F_{f}$ has a sublinear dependence on the interface velocity $\dot{X}$. As a consequence, $F_{f}$ is expected to dominate the dynamics when $\dot{X}$ is sufficiently small. After a renormalization by the inertial term, eq. (2) yields

$$
\ddot{X}+\omega_{0}^{2} X=-2 \sigma \omega_{0} \dot{X}-\alpha \omega_{0}^{4 / 3} R^{1 / 3} \dot{X}|\dot{X}|^{-1 / 3},
$$


where $\omega_{0}$ is the angular frequency scaling as $\omega_{0} \sim \sqrt{g / R}$, and $\sigma$ is the viscous damping coefficient which is proportional to $\sigma \sim \sqrt{\nu} /\left(R^{3 / 4} g^{1 / 4}\right)$. These scaling arguments are in line with the theoretical results obtained using first principles ${ }^{1,3}$. The last term on the right hand side is the nonlinear damping associated with the foam layer, where the prefactor $\omega_{0}^{4 / 3} R^{1 / 3}$ results from the renormalization, and $\alpha$ is the dimensionless coefficient of sublinear damping, which scales as $\alpha \sim h d^{-1} \gamma^{1 / 3} \mu^{2 / 3} \rho^{-1} R^{-5 / 3} g^{-2 / 3}$.

\section{B. Asymptotic solution and comparison to experiments}

In the limit of negligible capillary effects, $\alpha=0$, equation (3) reduces to a linearly damped harmonic oscillator. This equation is traditionally used to model the free-surface oscillations of a liquid in a tank ${ }^{2}$, with solution:

$$
X(t)=X_{0} e^{-\omega_{0} \sigma t} \cos \left(\sqrt{1-\sigma^{2}} \omega_{0} t\right),
$$

where $X_{0}$ is the initial amplitude. This expression is consistent with the experimental data reported in Fig. 2 for $h=0$ (no foam).

With foam and thus $\alpha \neq 0$, eq. (3) does not have a closed-form analytical solution. However, an asymptotic solution can be obtained by using the method of multiple scales as detailed in Nayfeh ${ }^{10}$. In the limit of small damping, $\sigma, \alpha \ll 1$, the solution of eq. (3) is asymptotic to $X(t) \sim a(t) \cos \left(\omega_{0} t\right)$, where the sinusoidal term is the solution of the undamped oscillator ( $\sigma=\alpha=0$ as in the leading order problem in the asymptotic scheme). The slow varying envelope $a(t)$ is determined at the next order by imposing a compatibility condition that involves only the right hand side terms in eq. (3) oscillating at the natural frequency of the system $\omega_{0} . X(t)$ reads:

$$
X(t)=\underbrace{\left[X_{0}^{1 / 3}-\frac{\alpha}{\sigma} c R^{1 / 3}\left(e^{\omega_{0} \sigma t / 3}-1\right)\right]^{3} e^{-\sigma \omega_{0} t}}_{a(t)} \cos \left(\omega_{0} t\right) .
$$

See supplemental material ${ }^{11}$ at [URL] for a detailed derivation of the asymptotic solution and the definition of the constant $c$ that is the Fourier coefficient of the Fourier component of $\dot{X}|\dot{X}|^{-1 / 3}$ synchronized to the natural frequency of the system $\omega_{0}$. Note that the frequency shift $\sqrt{1-\sigma^{2}}$ in eq. (4), is not present in the asymptotic solution (5) as it belongs to a higher order.

The asymptotic solution (5) to the sublinear model (SLM), is now compared and fitted to our experimental results. In Figure 2(b) the coefficients $\omega_{0}$ and $\sigma$ are set to their reference values obtained experimentally in the $h=0$ limit $\left(\omega_{0}=22.00 \mathrm{rad} / \mathrm{s}, \sigma=9.8 \cdot 10^{-3}\right)$. The sublinear coefficient $\alpha$ is thus the only unknown parameter of the model. Here, $\alpha$ is determined independently for each of the $h \neq 0$ cases by fitting the logarithmic decrement with equation (5). We find that our model captures the concave aspect of the linear decrement measured from experiments. Additionally, the larger the film thickness, the stronger the nonlinear damping coefficient. More precisely, our data in Fig. $2 B$ shows that the damping coefficient $\alpha$ increases linearly with the foam thickness $h$, further validating the proposed physical picture: in the range of thin foam layers investigated, the foam acts as collection of Plateau borders, which all equally contribute to the effective dissipative force. The number of contact lines, earlier estimated as the foam thickness to bubble diameter ratio $h / d$, is thus the relevant scale to evaluate $\alpha$.

\section{Sublinear friction and its finite-time arrest}

Assured that the reduced model (3) and its solution (5) are a quantitative representation of the sloshing dynamics with foam, we turn to examine the mechanistic impact of 

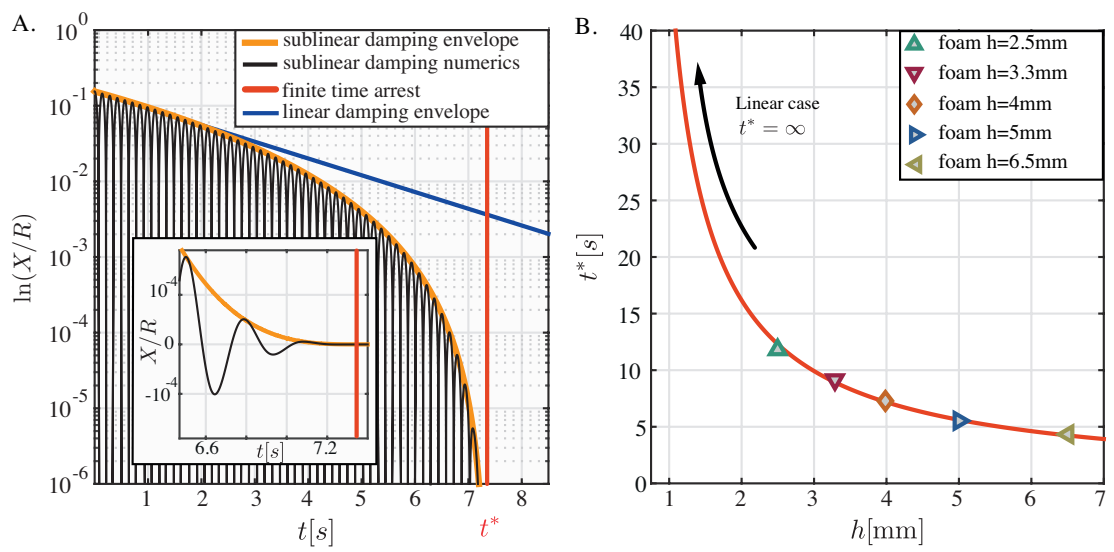

FIG. 4. (A) Numerical nonlinear solution of eq. (3) for $h=4 \mathrm{~mm}$ (black line), together with its envelope obtained by asymptotic analysis (orange line). The vertical red line indicates the finitetime of arrest $t^{*}$. The blue line represents the envelope of the linear model solution starting from the same initial condition. (B) Time of arrest as a function of the foam height $h$ for a single initial condition (from eq.(6) with $\alpha(h)$ reported in Fig. 2B). Symbols correspond to the values deduced from the experiments.

the sublinear damping introduced in our model. In Fig. $4 A$ we report the sublinear model (SLM) envelope $a(t)$ in the particular, yet representative, case $h=4 \mathrm{~mm}$. Its concave down behavior contrasts with the classical exponential relaxation adopted by previous works ${ }^{4}$, indicated by the blue line. This effect is due to an increase of the relative importance of sublinear friction forces compared to the inertial and restoring terms as the amplitude of oscillation decreases. The dominance of interfacial effects is induced by their sublinear dependance in the oscillating velocity. In fact, they diverge in the limit of vanishing oscillations, yielding a finite-time singularity. For the sake of the validation of our asymptotic solution (5), we report also in Fig. $4 A$ the numerical solution of eq. (3) obtained using a third order Runge-Kutta scheme.

Upon examination of the behavior of eq. (5) at very small amplitudes, we find the amplitude of sloshing to be exactly nil for time $t=t^{*}$, where $t^{*}$ is the time of arrest. This behavior contrasts the classical linear theory giving rise to an exponential decay for which $t^{*}=\infty$. In our model, $t^{*}$ is finite and using eq. (5), we can derive its analytical expression:

$$
t^{*}=\frac{3}{\sigma \omega_{0}} \ln \left[1+c^{-1} \frac{\sigma}{\alpha(h)}\left(\frac{X_{0}}{R}\right)^{1 / 3}\right] .
$$

In Fig. $4 B$ we show the variation of $t^{*}$ with $h$ and report the values corresponding to our experiments (as deduced from the fit of our experimental data using the SLM). The time of arrest $t^{*}$ diverges in the limit where $h=0$, as expected since this case corresponds to an exponential relaxation in our theoretical framework (see eq. (4)). A small foam layer $(h=2.5 \mathrm{~mm})$ is sufficient to bring this time to a finite and relatively small value $\left(t^{*} \simeq 12 \mathrm{~s}\right)$. Other values of $t^{*}$ corresponding to larger values of $h$ are of the same order (the smallest is $5 \mathrm{~s})$ and decrease if the foam thickness $h$ increases.

\section{CONCLUSIONS}

In our experiments we found that adding foam to a liquid dramatically damps its oscillatory motion but hardly affects its sloshing frequency. Without foam, sloshing is well approximated by a linearly damped harmonic oscillator relaxing exponentially with characteristic time $t_{\mu}=1 /\left(\sigma \omega_{0}\right)$, thereby predicting an arrest of motion for $t^{*}=\infty$. In contrast, owing to its cellular structure, foam introduces sublinear capillary forces, which, in turn, 
lead to a finite-time arrest of the fluid's motion. Equation (6) provides the expression for the time of arrest $t^{*}$, highlighting the importance of the ratio between the viscous damping $\sigma \sim \nu^{1 / 2} g^{-1 / 4} R^{-3 / 4}$ and the sublinear damping $\alpha \sim h d^{-1} \gamma^{1 / 3} \mu^{2 / 3} R^{-5 / 3} g^{-2 / 3} \rho^{-1}$ coefficients. The time of arrest $t^{*}$ is negatively correlated to $\alpha / \sigma$ and diverges when this ratio is small (corresponding to a linearly damped oscillator). Specifically, an increase in $h$, an increase in $\gamma$ or a decrease in $d$, all yield a decrease in $t^{*}$. The scaling argument for the sublinear coefficient $\alpha$ is in good agreement with the experimental measurements for various foam height $h$, see section III B. On the other hand, its dependence on the fluid density, $\rho$, its viscosity, $\mu$, and surface tension, $\gamma$, along with the dependence on the container size, $R$, and the foam bubble diameter, $d$, can in principle be probed experimentally by using different fluids, container size and foams and it is a future perspective of this work.

Note that this expression is only valid for small but finite foam layers. Sloshing with a large amount of foam may lead to the situation where only a fraction of the total volume of foam is effectively in motion. The upper part of the foam layer remains still, hence does not participate to the sublinear damping examined in this work. We therefore anticipate that $\alpha(h)$ does not remain linear for arbitrarily large values of $h$, but instead saturates, in turn giving rise to a plateau for $t^{*}$ in the limit of large foam thicknesses.

Similarly, the case $d=0$ and $h=0$ is ill-defined in our model. For these values, corresponding to a simple liquid-air interface, a liquid meniscus forms at the container walls. During sloshing, this advancing and receding contact line will generate a small, yet nonlinear, friction force ${ }^{5}$, which affects the damping rate measured in the experiments ${ }^{2,8}$. Evidence of these effects is apparent at very small amplitude as highlighted by the shaded area in Fig. $2 A$ for pure water. Although the accuracy of the measurements does not allow for a systematic analysis at this stage, this observation suggests that our work could generalize to a single moving contact line. Accounting for those effects - without foam to exacerbate them - requires a realistic contact line model ${ }^{2}$, with features such as contact angle hysteresis and the variations of contact angle with the contact line velocity.

Finally, we note that finite-time arrests are commonly observed in mechanical systems involving solid friction as a consequence of Coulomb's law, but have not frequently been reported in fluidic systems. Their importance in practical situations such as Euler's disk has been the subject of investigations in recent years ${ }^{12}$. Here, we have demonstrated that the effect of pure fluid dissipation in sub-millimetric menisci at a contact line is sufficient to bring a body of fluid to rest in finite time albeit the fluid's volume is significantly larger than that of the meniscus.

Financial support by ERC grant SimCoMiCs 280117 is gratefully acknowledged.

The authors warmly thank Dr Gabs brewery for their inspiring foam, and for providing the fuel for our experiments.

${ }^{1}$ H. Lamb, Hydrodynamics (Cambridge University Press, 1932).

${ }^{2}$ R. A. Ibrahim, Liquid Sloshing Dynamics: Theory and Applications (Cambridge University Press, 2005).

${ }^{3}$ K. Case and W. Parkinson, "Damping of surface waves in an incompressible liquid," J. Fluid Mech. 2, 172-184 (1957).

${ }^{4}$ A. Sauret, F. Boulogne, J. Cappello, E. Dressaire, and H. A. Stone, "Damping of liquid sloshing by foams," Phys. Fluids 27, 022103 (2015).

${ }^{5}$ I. Cantat, "Liquid meniscus friction on a wet plate: Bubbles, lamellae, and foams," Phys. Fluids 25, 031303 (2013).

${ }^{6}$ F. Bretherton, "The motion of long bubbles in tubes," J. Fluid Mech. 10, 166-188 (1961).

${ }^{7}$ N. D. Denkov, V. Subramanian, D. Gurovich, and A. Lips, "Wall slip and viscous dissipation in sheared foams: Effect of surface mobility," Colloids Surf. A 263, 129-145 (2005).

${ }^{8}$ B. Cocciaro, S. Faetti, and M. Nobili, "Capillarity effects on surface gravity waves in a cylindrical container: wetting boundary conditions," J. Fluid Mech. 231, 325-343 (1991).

${ }^{9}$ L. Landau and B. Levich, "Dragging of a liquid by a moving plate," Acta Phys. USSR 17, 42 (1942).

${ }^{10}$ A. H. Nayfeh, Perturbation Methods (John Wiley \& Sons, 2008).

${ }^{11}$ See Supplementary Material at [URL] for details about the asymptotic analysis (Section III),.

${ }^{12}$ H. K. Moffatt, "Euler's disk and its finite-time singularity," Nature 404, 833-834 (2000). 